\title{
Laser Gated Viewing: an enabler for Automatic Target Recognition?
}

\author{
Ernst Bovenkamp ${ }^{\mathrm{a}}$, Klamer Schutte ${ }^{* \mathrm{~b}}$ \\ ${ }^{a}$ TNO Science and Industry, Stieltjesweg 1, Delft, The Netherlands; \\ ${ }^{\mathrm{b}}$ TNO Defence, Security and Safety, Oude Waalsdorperweg 63, The Hague, Netherlands
}

\begin{abstract}
For many decades attempts to accomplish Automatic Target Recognition have been made using both visual and FLIR camera systems. A recurring problem in these approaches is the segmentation problem, which is the separation between the target and its background.

This paper describes an approach to Automatic Target Recognition using a laser gated viewing system. Here laser-flash illumination is used in combination with a gating viewer such that only a small part in the distance domain is seen in a single image. In our approach, using an Intevac LIVAR 4000 imaging system, we combined several images with different gate settings to construct a $3 \mathrm{D}$ data cube. This paper describes the preprocessing and filtering steps taken to obtain a range image for which pixel values represent the distance between camera and objects in the illuminated scene. Depth segmentation is performed using the global histogram of this range image. After this depth segmentation very good 2D object segmentations can be obtained which can be used to classify persons and vehicles. An outlook will be given towards operational application of this procedure.
\end{abstract}

Keywords: Laser Gated Viewing, Automatic Target Recognition

\section{INTRODUCTION}

For many years Automatic or Aided Target Recognition (ATR) is pursued for Target Acquisition using visual or FLIR cameras. The aim is to support the human in detection and classifying possible targets. However, the task of discriminating between clutter and objects is difficult, and often a human operator will outperform a dedicated automated system. We expect a gated viewing system can make the difference: by exploiting its range dependent return one can exploit the 3D difference between targets and random clutter. Most notably, object segmentation is an essential yet erroneous process in many ATR approaches, where foreground-background segmentation often is rather simple using gated viewing,

Laser Illuminated Viewing and Ranging (LIVAR) is an active imaging technique which uses a pulsed-laser to illuminate a scene in combination with a gating camera. The camera has a very fast electronic shutter which operates such that only reflected light from within a particular distance or range gate is recorded. By shifting the range gate one can obtain images from different depths. These images are combined into a data cube from which the distance between camera and objects in the illuminated scene can be derived. This yields a so called 'range image' from which the 3D scene can be reconstructed.

Because the gated viewer is an active system, it is in particular an excellent night-vision system. There is in principle the risk of detection by others, however, the 1.5 micron laser radiation of the Intevac viewer is hard to detect. A thermal imager is not sensitive enough to detect the minimal temperature increase when the beam hits the target. Further, image intensifiers are only sensitive at the near IR spectral range and not at the short IR spectral range around 1.5 micron.

The identification potential of the gated viewer is based on the high resolution of the short wavelength of 1.5 micron compared to the long wavelength range of thermal imagers. A very long focal length is possible without extreme aperture sizes due to the active illumination. In addition, range information is provided so that the size of the target can be accurately estimated. By separating the target from foreground and background using the range gate, it is much easier to use ATR. The active imaging helps in looking through mist, smoke, obscurants, and camouflage. In particular, it is often possible to look into cars and rooms through the window glass. Another important feature is the reduction of the

\footnotetext{
*klamer.schutte@tno.nl; phone +31 70374 0469; fax +31 70374 0653; www.tno.nl

Laser Radar Technology and Applications XV, edited by Monte D. Turner, Gary W. Kamerman, Proc. of SPIE Vol. 7684, 76840Z · (c) 2010 SPIE · CCC code: 0277-786X/10/\$18 · doi: 10.1117/12.849644
} 
influence of parasitic light such as the path radiance, scatter, sun and car lights [1]. Note that in theory a gated viewing based system should outperform a range profiling system [9], as it has additional access to spatial information.

Section 2 describes the laser gated viewing system and scene used in the experiments. Section 3 describes methods to retrieve distance measurements from sets of laser gated images. In Section 4 both 2.5D and 3D methods are given which aim to segment the illuminated scene into meaningful objects. Section 5 shows classification of these objects into vehicles and humans and Section 6 describes a possible operational scenario using laser gated viewing. Finally, in Section 7 conclusions are given.

\section{EXPERIMENTAL SETUP}

The imaging system used was an Intevac LIVAR 4000 system. The laser consists of a flashlamp pumped OPO shifted ND:YAG laser which radiates pulses of 1.5 micron with a width of $10 \mathrm{~ns}$. The Intevac SWIR camera has a TE-EBCMOS sensor which takes 10 bit images of $640 \times 480$ pixels and has $6.1 \mathrm{mrad}$ horizontal and $4.6 \mathrm{mrad}$ vertical field of view. The minimum gate width setting was $150 \mathrm{~ns}$ which represents a distance range of 22.5 meters.

The system was pointed at various locations in the scene as shown in Figure 1 in stable daylight conditions with sunny weather and virtually no overcast. The distance of the scene was approximately 780 meters. The camera gate was set at the minimum width of 22.5 meters and was shifted with a minimum gate shift of 1 meter from 765 to 800 meter. For each gate setting 10 images were taken. So for each of the 35 range settings 10 images were taken, which resulted in 350 images for the scene.

For each range setting the mean of 10 images was taken to reduce target and atmospheric influences. As the system turned out to have floating input column differences, this was compensated for. Also, defective pixels were repaired by taking the mean of its neighbors.

A 3D data cube was then constructed from the set of mean images which resulted in a data cube of $640 \times 480 \times 35$ pixels.

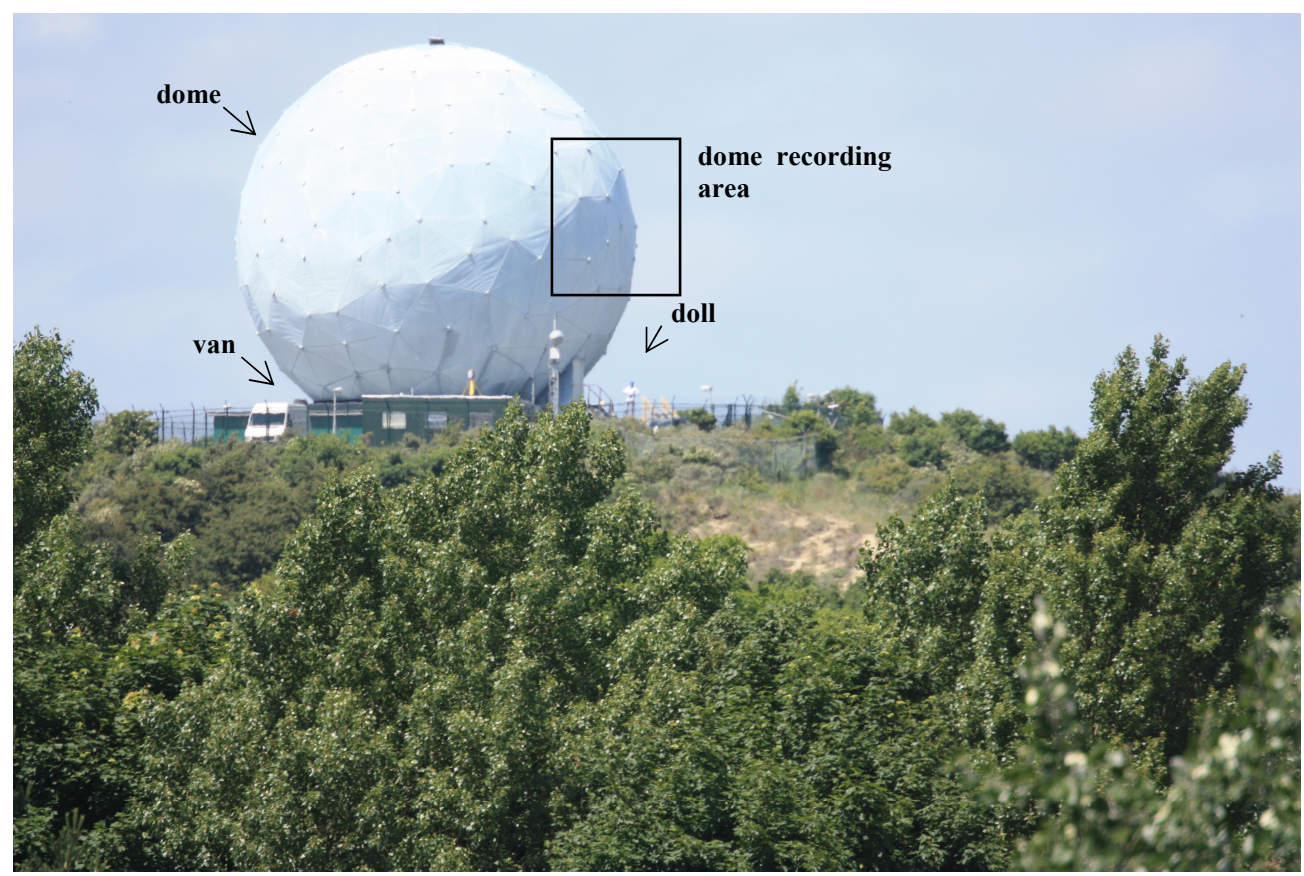

Figure 1. Overview of the testing area with radar dome, armed doll to the lower right and van to the lower left of the dome. The dome recording area is used to determine the laser range gate profile. 


\section{DEPTH RETRIEVAL}

In the 3D data cube each $(x, y, z)$ position represents the returned signal $s_{x y}(z)$ at image location $(x, y)$ with range setting $z$. As the gate is shifted with a constant distance of 1 meter, $z$ is proportional to the distance. So $z=0$ represents the first gate distance setting which was at 765 meter in the experiment described below.

To obtain depth information from the 3D data cube one could take the signal $s_{x y}(z)$ and sample a single position on the flank of $s_{x y}(z)$ and one around its maximum and use these to match with the gate profile as is done in [2]. However, we found the returned signal to be too noisy for this method to be robust. A more robust method was to take the complete signal $s_{x y}(z)$ and convolve it with the gate profile $g(z)$ which results in the fitness function $c(k)$. The maximum of $c(k)$ then serves as the first rough distance estimate $d_{0}$ of an object in location $(x, y)$. The discrete convolution of gate $g(z)$ with signal $s_{x y}(z)$ as measured in an image location $(x, y)$ is determined by:

$$
c(k)=\sum_{j} g(j) s_{x y}(k+1-j)
$$

and the first estimate of the distance $d_{0}$ of some object in location $(x, y)$ is determined by:

$$
d_{0}=\underset{k}{\arg \max } c(k)
$$

To obtain submeter precision the shape of $c(k)$ was also taken into consideration. Here the most common technique is to fit a parabola to the values of $c(k)$ for the initial distance estimate $\mathrm{d}_{0}$ and its immediate neighboring points $d_{-l}$ and $d_{l}$ and then to take the minimum of the fitted parabola as the new distance estimate $d_{s}$ which then has submeter accuracy. This method is most efficient in terms of accuracy achieved versus computational effort [3]. In the remainder this method will be called the quadratic method and is formally described as:

$$
d_{s}=d_{0}+\frac{\left(c\left(d_{-1}\right)-c\left(d_{1}\right)\right)}{2\left(c\left(d_{-1}\right)-2 c\left(d_{0}\right)+c\left(d_{1}\right)\right)}
$$

Another method, which will be called the differential method, takes the first derivative of $c(k)$ to calculate $d_{s}$ and is claimed to have high performance especially for wider peaks of $c(k)$. In addition it is said to show good results at high noise levels where the detection error of other methods starts to increase [4]. The first derivative of $c(k)$ is approximated with:

$$
\bar{c}(k)=c(k+1)+c(k+2)-c(k-1)-c(k-2)
$$

The derivative of $\bar{c}(k)$ crosses the $\mathrm{x}$-axis and the discrete points closest to this crossing are called $\left(x_{0}, y_{0}\right)$ and $\left(x_{1}, y_{1}\right)$. The submeter accurate distance estimate $d_{s}$ of an object in $(x, y)$ is now calculated as:

$$
d_{s}=x_{0}-y_{0} * \frac{x_{1}-x_{0}}{y_{1}-y_{0}}
$$

Both methods were tested and yielded similar results although the quadratic method had a somewhat lower variance, whereas the differential method offered somewhat more detail in the object distance measurements. In the remainder of this article we will use the quadratic method unless stated otherwise.

\subsection{Gate profile estimation}

The gate profile used was retrieved by pointing the gated viewer to the dome recording area and measuring the average returned signal in various locations where the dome was relatively flat and homogenous. The shape of this general signal is shown in Figure 2. From this graph we concluded that the shape of the gate profile can be described by an asymptotic signal growth from right to left, followed by an asymptotic signal decay. Both signal growth and decay are most likely explained by an RC-type gate sensitivity behavior. 


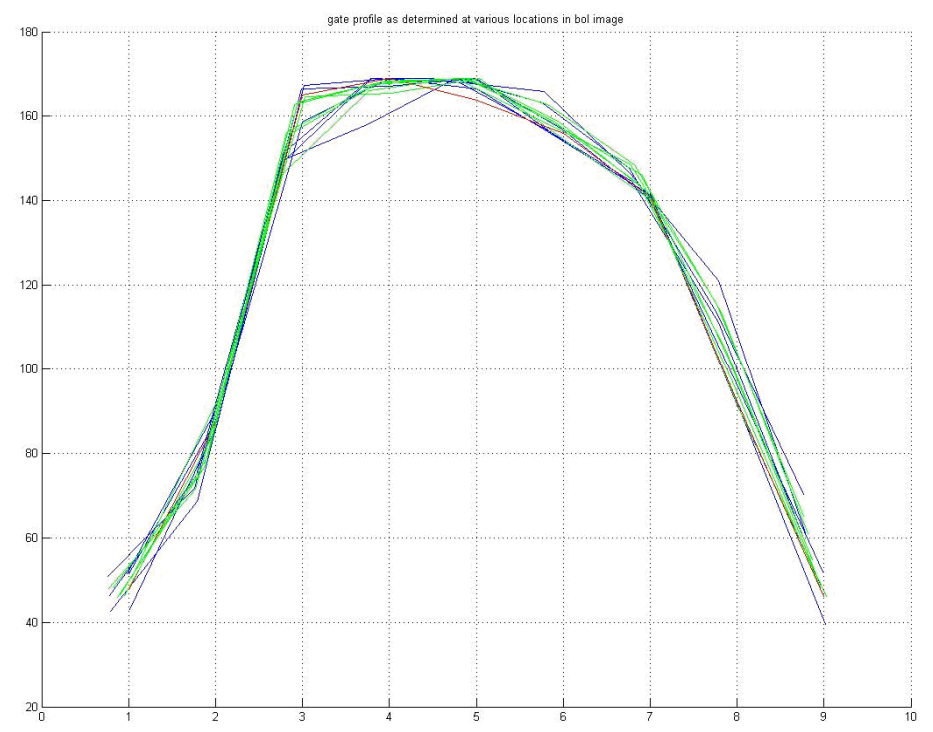

Figure 2. Gate profiles extracted from the dome recording area as shown in Figure 1. The gates are scaled on the maximum value and repositioned horizontally. Each x-position represents a distance of 3 meters.

The gates profiles as shown in Figure 2 were retrieved from 6 square regions and 7 lines in the image. As the retrieved gate profile might have a significant effect on the subsequent distance measurements, the influence of these gate profiles was tested with distance measurements on relatively flat areas perpendicular to the viewing direction and obtained from a number of different scenes and experiments. Generally a standard deviation of the distance of 0.02 meter was measured using different gate profiles. This is well below the average standard deviation of object range which was measured around 0.20 meters. Otherwise the relatively low standard deviation also shows the robustness of the distance measuring method to the gate profile and robustness of the gate profile retrieval process. As no significant difference was found between gate profiles, one single profile was selected and used to obtain range measurements in the rest of this paper.

In the experiments we used 10 images per gate distance, and 1 meter gate shifts, leading to a depth accuracy up to $0.2 \mathrm{~m} /$ pixel. Experiments using fewer images showed to provide less accurate depth estimates.

Retrieval of depth information from the 3D data cube by convolving the scene with the gate profile $g(k)$ and applying the quadratic submeter method results in the range image $I_{R}$ of which an example is shown in Figure $3 \mathrm{~b}$.
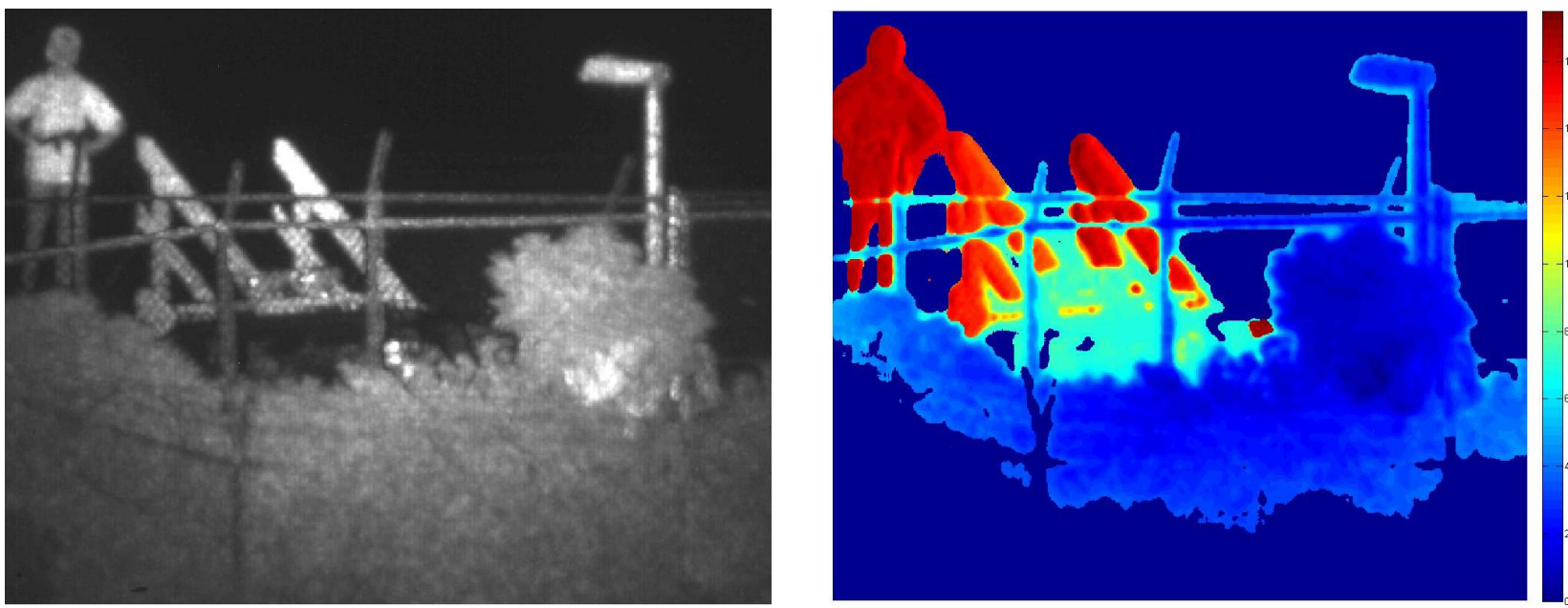

Figure 3. (a) Maximum projection of illuminated scene at approximately 780 meters and (b) measured distance from the 3D data cube using the quadratic subpixel method with filtering of low reliability pixels. 


\section{DEPTH SEGMENTATION}

The range image $I_{R}$ as shown in Figure $3 \mathrm{~b}$ is merely a cloud of $(x, y$,distance) points. In order to retrieve objects from the range image we investigated both a $2.5 \mathrm{D}$ and $3 \mathrm{D}$ approach.

\subsection{5D image segmentation}

In the $2.5 \mathrm{D}$ approach the range image $I_{R}$ is first segmented into interesting distance ranges. This is done automatically by analyzing the histogram of $I_{R}$ as shown in Figure 4a. The figure shows potentially interesting objects around ranges of 2.5, 7.5 and 16.0 meters for this particular experiment. Range intervals are calculated from the histogram, but are restricted to be maximally $+/-3$ meters from the peak value. This is done to prevent too large intervals of interest when no objects are present. Relative range intervals automatically calculated for this example were [0.5 4.0], [6.0 9.0], and [14.5 19.0] meters. Further peak values were set to be at least 2 meters apart.
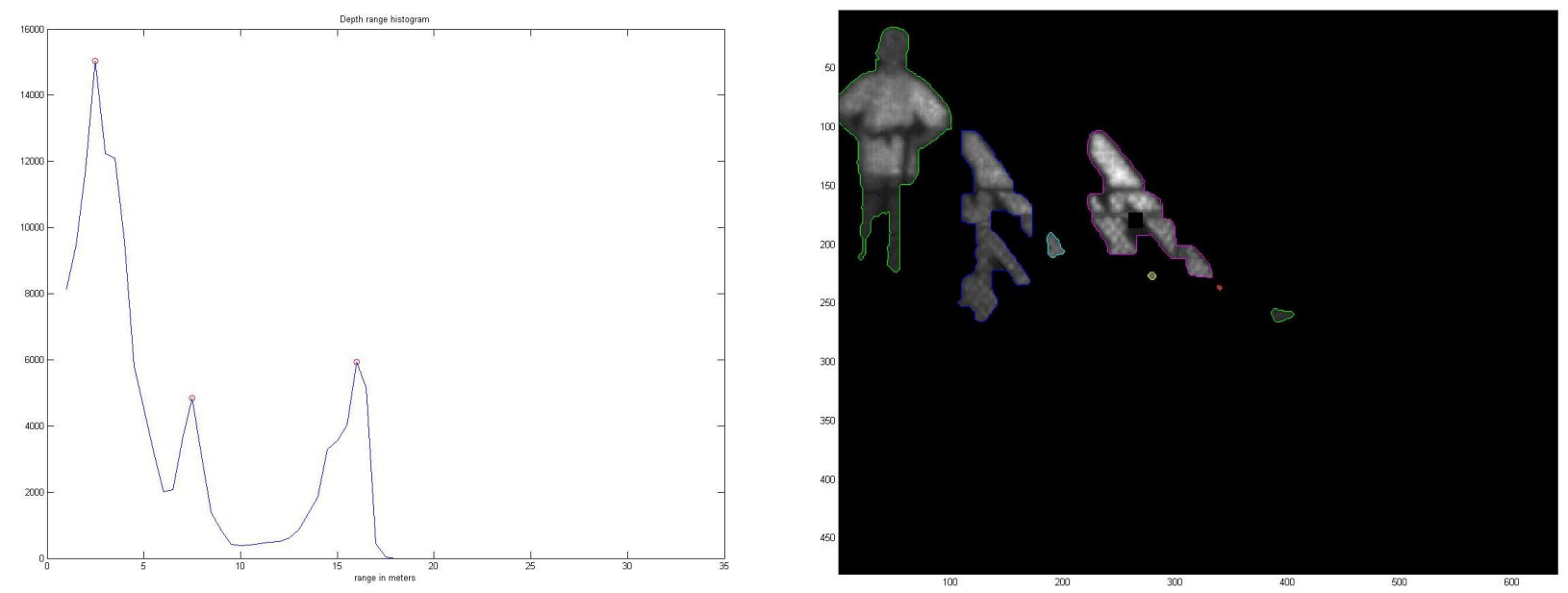

Figure 4. (a) Histogram of range image $I_{R}$ and automatic selection of interesting distance ranges $r_{i}$. (b) Automatically segmented objects at a relative distance between 14.5 and 19.0 meters.

The next step is to create binary object images $I_{r i}$ from the range image $I_{R}$ for each of the ranges $r_{i}$. Pixels in the binary object image $I_{r i}$ are set according to the following formula:

$$
I_{r_{i}}(x, y)= \begin{cases}1 & \text { iff } \quad l b\left(r_{i}\right) \leq I_{R}(x, y) \leq r b\left(r_{i}\right) \\ 0 & \text { otherwise }\end{cases}
$$

where $l b\left(r_{i}\right)$ is the left boundary and $r b\left(r_{i}\right)$ is the right boundary of range $r_{i}$. The aim of this step is to separate objects in the image which are at different distance ranges from the camera.

The resulting binary images $I_{r i}$ consist of blobs with holes which may be parts of one object due to partial occlusion in the foreground. On these images simple morphological operations are applied with the objective to grow blobs together which belong to the same object, while preventing blobs of different objects to merge. The basis procedure used is:

1. Determine the foreground of $I_{r i}$ to detect potential occlusion of objects in $I_{r i}$.

2. Remove small objects from foreground and background to reduce noise.

3. Fill small holes in the background to reduce noise.

4. Do $N$ closings on the background and remove parts not occluded by the foreground.

This way a partially occluded object in the background may 'grow together' while separate objects remain separate. The result of the complete procedure when applied to the range image as shown in Figure $3 \mathrm{~b}$ for the distance range of 14.5 to 19.0 meters yields the object image as shown in Figure 4b. The figure shows that the legs of the doll are grown to its 
body despite occlusion by the fence, whereas doll and stairs are separated as individual objects. Otherwise the handrails of the stairs are largely grown together although also occluded by the fence.

\subsection{D image segmentation}

One of the possible flaws of 2.5D segmentation occurs when objects extend over a considerable distance in the direction of the line of sight. Also objects are condensed into a 2D projection while their 3D structure might be revealed from the data. Therefore also complete 3D segmentations of the image were made without making any assumptions about potential distance ranges. The range image $I_{R}$ was again taken as the point of departure, but rather then segmenting it into a number of $2 \mathrm{D}$ binary images a 3D mesh was fitted on the $(x, y$,distance) point cloud as represented by the range image $I_{R}$. To get the 3D mesh a crust based algorithm [5] was used. The result is shown in Figure 5a.
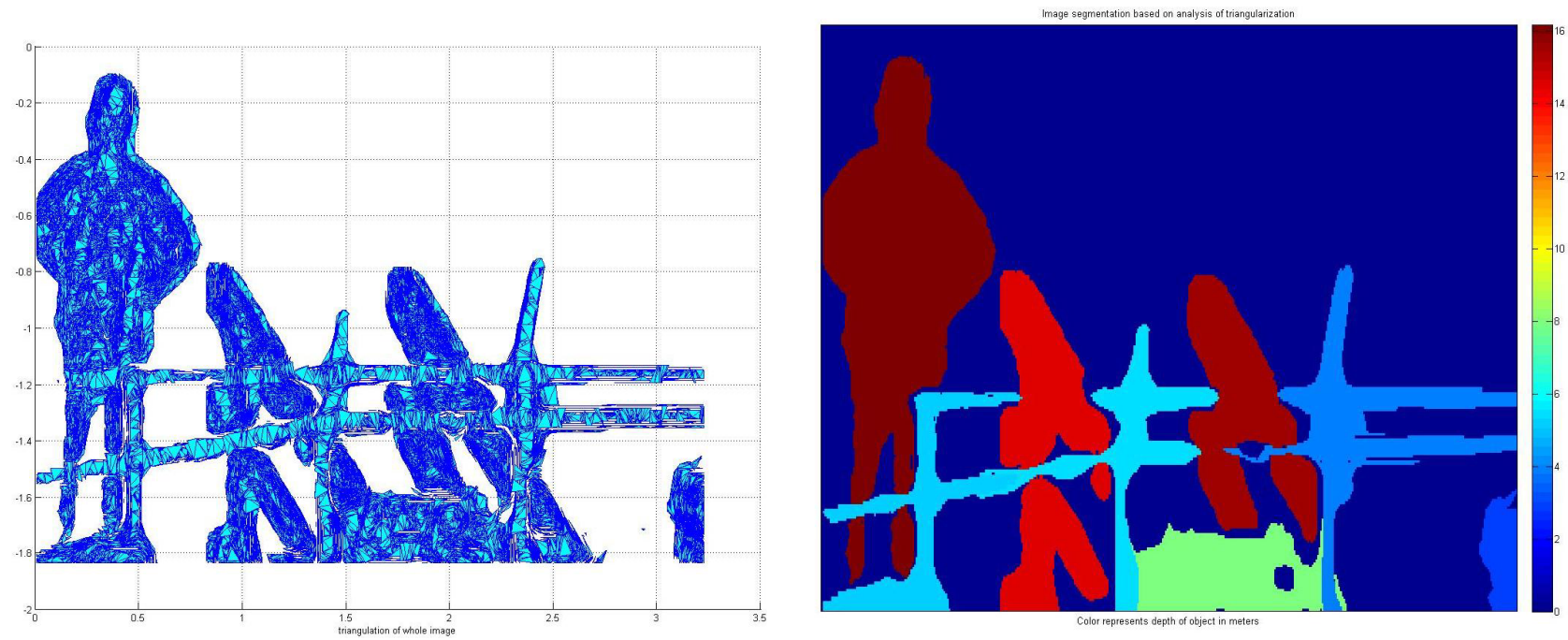

Figure 5 (a) 3D mesh of a part of the range image $I_{R}$ as shown in Figure 1b. (b) 2D projection of 3D objects after analysis and pruning of initial mesh. The color of each object represents its depth in meters. Objects in the background are occluded by objects in the foreground

The resulting 3D image mesh was analyzed in the following way:

1. Search the $3 \mathrm{D}$ mesh for faces with long edges. A face is removed when is has an edge with a length above a certain threshold. The threshold used was $0.08 \mathrm{~m}$ in $x y$-direction and $0.10 \mathrm{~m}$ in the $z$-direction.

2. Search the remaining edges for connected components. Connected components with more than 1000 edges are kept and considered to be objects in the scene.

The result of this procedure is a number of $3 \mathrm{D}$ objects. The $2 \mathrm{D}$ projection of these objects for the present example is shown in Figure $5 \mathrm{~b}$. The object color represents the relative mean distance of the object to the camera. The figure also shows that although the doll is occluded by the fence, it is segmented as one 3D object (same color). Similarly the handrails of the stairs are segmented as single solid 3D objects. The positioning of the objects in the scene is shown in Figure 6. 

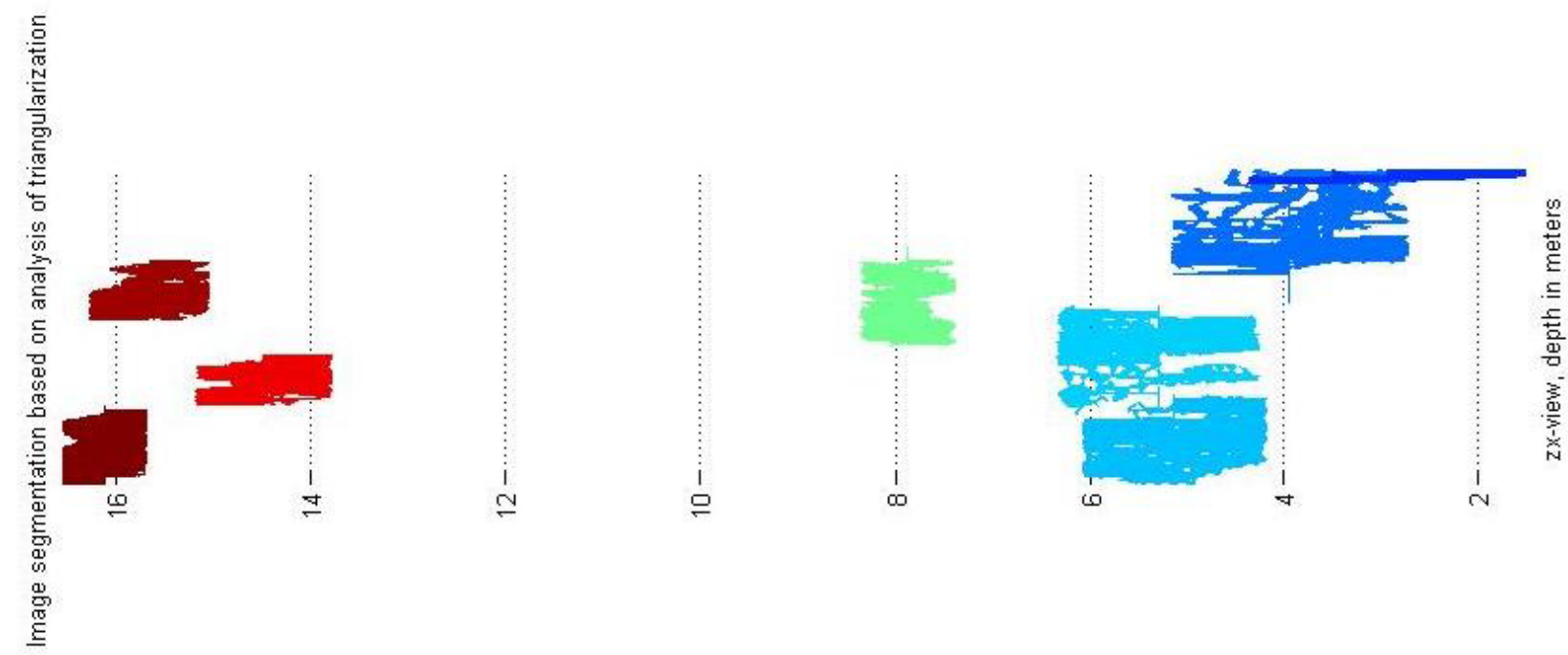

Figure 6. Positioning of the 3D objects in the illuminated scene using 3D crust based object segmentation.

\section{OBJECT SEGMENTATION AND CLASSIFICATION}

For each of the objects found with either the $2.5 \mathrm{D}$ or $3 \mathrm{D}$ approach general features like object dimensions, reflectivity, curvature, etc. were calculated. These features were used to see if humans and vehicles might potentially be classified automatically. For human detection we simply used height and width and for vehicle detection we inspected the dimensions and number of retro reflections on the object as these are often caused by the lights of a vehicle. This resulted in the detections as shown in Figure 7. As only a limited data set was available, the results are only preliminary and have no solid statistical foundation. However, it does show that it is at least potentially possible to automatically detect humans and vehicles at very large distances using a laser gated viewing approach.
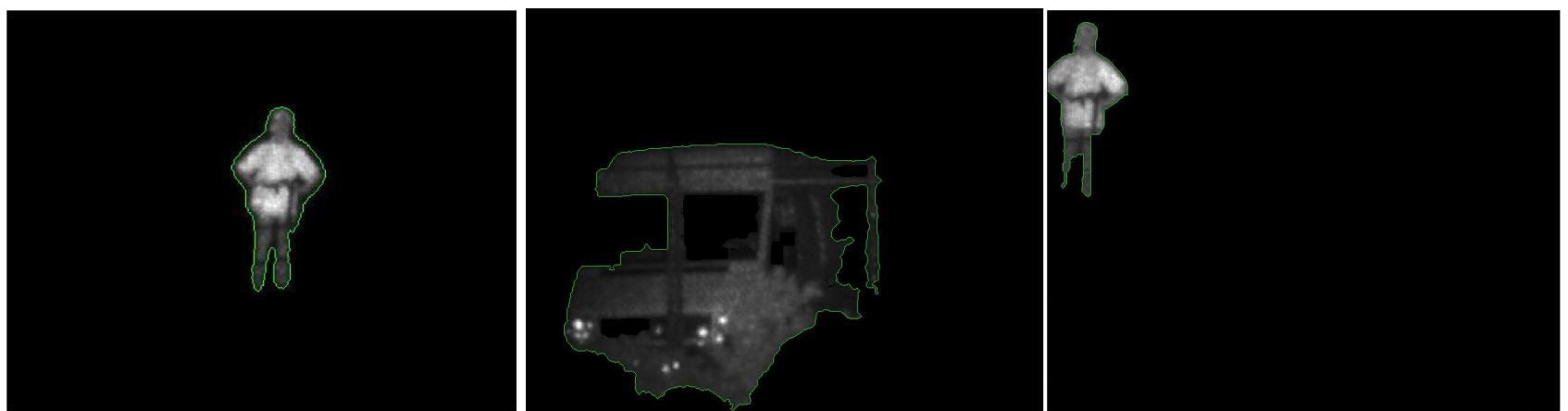

Figure 7. Automatically classified objects using the 2.5D approach with classification based on object dimensions and retro reflections.

\section{OPERATIONAL USE: FUTURE SYSTEM REQUIREMENTS}

The current experimental setup uses 35 range gates with 10 images each. In its operation every range gate has to be adapted by hand, leading to a long acquisition time. With its frame rate of close to $10 \mathrm{~Hz}$, we possibly could acquire the total data set after about 35 seconds after an automation of the range adaption step. This would make application a viable option for stationary targets, especially after a possible optimization w.r.t. to the total amount of frames required. Alternatively, one should use a LADAR system dedicated for per-pixel range acquisition [6,7], leading to acquisition times compatible with dynamic targets.

A gated viewing system provides good imaging and excellent recognition capabilities. For many applications it needs to be supplemented by a search sensor for initial target acquisition, in effect being part of a multi-sensor system [8]. 
More experiments are necessary to rigorously test the object classification process. Next to the present feature based classification (3D) model based classification may also be a promising approach because object knowledge can then be used to deal with partial occlusion of objects by the foreground. Otherwise we are planning more experiments to determine the exact depth resolution of laser gated viewing under various circumstances including reflective surfaces.

\section{CONCLUSIONS}

In this paper it was demonstrated that laser gated viewing does in principle support automated target recognition. Convolving a general gate profile with the $3 \mathrm{D}$ data cube obtained from an illuminated scene allows distance measurements with a standard deviation of $0.20 \mathrm{~m}$ or less which are summarized in a range image. In combination with automatic scene separation in distance ranges or by directly analyzing a 3D mesh fitted to the range image, objects can be separated from the background and occlusions can partially be repaired. These 2D or 3D objects can than be classified into objects of interest like humans and vehicles. The use of depth information as made available by a laser gated viewing significantly eases this recognition process, effectively acting as the enabler for ATR.

\section{REFERENCES}

1. E. Repasi, P. Lutzmann, et al. "Advanced short-wavelength infrared range-gated imaging for ground applications in monostatic and bistatic configurations”. Applied Optics, Vol. 48, Issue 31, pp. 5956-5969, 2009.

2. D. Monnin, A.L. Schneider, F. Christnacher and Y. Lutz. "A 3D Outdoor Scene Scanner Based on a Night-Vision Range-Gated Active Imaging System", Third international Symposium on 3D Data Processing Visualization and Transmission (3DPVT'06), pp. 938-945, 2006.

3. B. Cyganek and J. P. Siebert. "An introduction to 3D Computer Vision Techniques and Algorithms", J. Wiley \& Sons, 2009

4. J. Forest, J.M. Teixidor, J. Salvi, E. Cabruja. "A Proposal for Laser Scanners Sub-pixel Accuracy Peak Detector". Workshop on European Scientific and Industrial Collaboration, pp 525-532, Mickolc (Hungria) May 28-30, 2003.

5. N. Amenta, M. Bern, and M. Kamvysselis. "A new Voronoi-based surface reconstruction algorithm". Proc. of the 25th annual conference on Computer graphics and interactive techniques, pp 415-421, 1998.

6. Ian Baker, Daniel Owton, Keith Trundle, Peter Thorne, Kevin Storie, Philip Oakley and Jeremy Copley, “Advanced infrared detectors for multimode active and passive imaging applications", Infrared Technology and Applications XXXIV, Proc. of SPIE Vol. 6940 (2008)

7. Roger Stettner, Howard Bailey and Steve Silverman. "Large format time of flight focal plane detector development", Laser radar technology and applications X, Proceedings of the SPIE Vol. 5791 (2005)

8. A. C. van den Broek, S. P. van den Broek, J. C. van den Heuvel, P. B. W. Schwering, and A. W. P. van Heijningen, “A multi-sensor scenario for coastal surveillance", Proceedings of the SPIE Vol. 6736 (2007)

9. van den Heuvel, J.C. and Pace, P. and Bekman, H.H.P.T. and van Putten, F.J.M. and Schleijpen, R.M.A., "Experimental validation of ship identification with a laser range profiler", Proceedings of the SPIE Vol. 6950 (2008) 